\title{
ON DISCONJUGACY CRITERIA
}

\section{PHILIP HARTMAN ${ }^{1}$}

1. Disconjugacy criteria. In the differential equation,

$$
x^{(n)}+p_{0}(t) x^{(n-1)}+\cdots+p_{n-2}(t) x^{\prime}+p_{n-1}(t) x=0,
$$

let the coefficients be real-valued and summable on an interval $I$. The equation (1.1) is said to be disconjugate on $I$ if no solution $x(t) \not \equiv 0$ has $n$ zeros, counting multiplicities, on $I$. The object of this note is to derive disconjugacy criteria, for a compact interval $I$ $=[a, b]$ of length $|I|=b-a$, generally related to conditions of the type

$$
\sum_{k=0}^{n-1} A_{k}|I| k\left\|p_{k}\right\| \leqq 1
$$

where $A_{0}, \cdots, A_{n-1}$ are constants and $\|\cdots\|$ is the $L^{1}(I)$ norm. For $n=2$, a known disconjugacy condition is

$$
2^{-1}\left\|p_{0}\right\|_{1}+2^{-2}|I| \cdot\left\|p_{1}^{+}\right\| \leqq 1,
$$

where $r^{+}=\max (0, r)$ and $r^{-}=\max (0,-r)$, and follows from Levin's condition [5],

$$
\left(\exp \frac{1}{2}\left\|p_{0}\right\|_{1}\right)\left\|p_{1}^{+}\right\| \leqq 4 /|I|,
$$

by virtue of $e^{-x} \geqq 1-x$ for $x \geqq 0$; cf. also [0]. If $p_{0} \equiv 0$, then (1.3) and (1.4) reduce to a condition of Lyapunov; e.g., [1, p. 346].

Introduce the constants

$$
C_{n}=C(n-1,[n / 2]) / n !,
$$

where $[r]$ is the largest in teger not exceeding $r$ and $C(n, j)=n ! / j !(n-j) !$ is the binomial coefficient. These numbers $C_{1}=1, C_{2}=1 / 2, C_{3}=1 / 3$, $C_{4}=1 / 8, \cdots$ appear in Levin [3], [4] and Hukuhara [2]. Hukuhara [2] gives a disconjugacy criterion related to (1.2) with

$$
A_{0}=1 \text { and } A_{k}=2^{-k} C_{k} \quad \text { for } k=1, \cdots, n-1 .
$$

Using his method, this will be sharpened to (1.12) below.

In [6], Nehari states that (1.2) is a disconjugacy criterion if

Received by the editors January 28, 1969.

1 Research partially sponsored by the Air Force Office of Scientific Research, Office of Aerospace Research, United States Air Force, under AFOSR Contract No. F44620-67-0098. 


$$
A_{k}=2^{-k-1} \quad \text { for } k=0, \cdots, n-1 .
$$

It has been noted by Nehari (see [0]) that his proof of this assertion is incorrect and that the validity of the assertion is undecided. It will be seen that Nehari's statement is correct and, in fact, is contained in (1.9) below. For, on the one hand, a simple calculation shows that $\left(C_{k} / 4\right)[k /(k+1)]^{k-1} \leqq 2^{-k-1}$ for $k=1,2,3$ and, on the other hand, it is easy to verify that $C_{k} / 4<2^{-k-1}$ if $k \geqq 4$. (In [7], Zaĭceva asserts a refinement of criterion (1.7), but her inequality (16) on p. 765 does not seem correct, and this invalidates her proof.)

I would like to thank Professor A. M. Fink for calling my attention to the question of the validity of the criterion (1.7) and for a helpful correspondence in the course of the preparation of this note.

THEOREM 1. The following are disconjugacy criteria for (1.1) on $I=[a, b]:$

(i) $p_{0}, \cdots, p_{n-1}$ satisfy

$$
\left(\exp \frac{1}{2}\left\|p_{0}\right\|\right) \sum_{k=1}^{n-1}\left(C_{k} / 4\right)[k /(k+1)]^{k-1}|I|^{k}\left\|p_{k}\right\| \leqq 1,
$$

in particular,

$$
\frac{1}{2}\left\|p_{0}\right\|+\sum_{k=1}^{n-1}\left(C_{k} / 4\right)[k /(k+1)]^{k-1}|I|^{k}\left\|_{p_{k}}\right\| \leqq 1 ;
$$

(ii) if, in addition, $p_{1}(t) \equiv \cdots \equiv p_{m-1}(t) \equiv 0$ for some $m, 2 \leqq m \leqq n-1$, then (1.8) can be relaxed to

$$
\exp \left(\frac{1}{2}\left\|p_{0}\right\|\right) \sum_{k=m}^{n-1}\left(C_{k} / 2^{m+1}\right)[k /(k+m)]^{k-m}|I| k\left\|p_{k}\right\| \leqq 1 ;
$$

(iii) $p_{0}, \cdots, p_{n-1}$ satisfy

$$
\begin{aligned}
\left(\exp \left\|p_{0}\right\|\right) & \left\{\sum_{k=1}^{n-1}\left[\left(C_{k} / 2^{k+1}\right)|I|^{k}\left\|p_{k}\right\|\right]^{2}\right. \\
+ & \left.\frac{1}{2} \sum_{j \neq k} \sum C_{j} C_{k} j^{j} k^{k}(j+k)^{-j-k}|I|^{j+k}\left\|p_{j}\right\| \cdot\left\|p_{k}\right\|\right\} \leqq 1
\end{aligned}
$$

(iv) the quantities $Q_{1}, Q_{2}$ satisfy

$$
\max \left(Q_{1}, Q_{2}\right) \leqq 1,
$$

where

$$
Q_{1}=\left(\exp \int_{a}^{(a+b) / 2} p_{0}^{+} d t\right) \sum_{k=1}^{n-1} C_{k} 2^{-k}|I|^{k} \int_{a}^{(a+b) / 2}\left|p_{k}\right| d t
$$




$$
Q_{2}=\left(\exp \int_{(a+b) / 2}^{b} p_{0}^{-} d t\right) \sum_{k=1}^{n-1} C_{k} 2^{-k}|I|^{k} \int_{(a+b) / 2}^{b}\left|p_{k}\right| d t
$$

2. The Levin inequalities. Theorem 1 will be deduced from the following inequalities given by Levin [4] and Hukuhara [3]. (The latter also makes the assumption $a \leqq a_{0} \leqq a_{1}$.)

Lemma $2.1_{n}$. Let $n \geqq 1, I=[a, b]$, and $x(t) \in C^{n}(I)$. Let $x(t)$, $x^{\prime}(t), \cdots, x^{(n-1)}(t)$ vanish at points of $I$, say

$$
x^{(k)}\left(a_{k}\right)=0 \quad \text { for } k=0, \cdots, n-1,
$$

and let

$$
a \leqq a_{1} \leqq \cdots \leqq a_{n-1} \leqq b .
$$

Then

$$
\max _{I}|x(t)| \leqq M C_{n}|I|^{n}, \text { where } M=\max _{I}\left|x^{(n)}\right|
$$

and $C_{n}$ is defined by (1.5). $C_{n}$ cannot be replaced by a smaller constant.

Levin's proof [4] uses some functional analysis and the KreinMilman theorem on extreme points of convex sets. Hukuhara's proof [2] is an elementary calculus proof. We shall give a different, short, elementary proof which will yield sharper inequalities (cf. (2.6)-(2.7), (2.8)-(2.9), (3.5) and (4.3) below) and has the following consequence:

CoROLlary 2.1. The assertion (2.3) in Lemma $2.1_{n}$ can be strengthened to

$$
\int_{a}^{b}\left|x^{\prime}(t)\right| d t \leqq M C_{n}|I|^{n}
$$

whether or not $x(t)$ has a zero $t=a_{0}$ on $I$.

REMARK. It is easy to verify that equality in (2.3) holds if $\left|x^{(n)}(t)\right|$ $\equiv M, a_{k}=a_{n-1}$ for $k \geqq n / 2, a_{k}=a_{0}$ for $k<n / 2$, and $a=a_{0}, b=a_{n-1}$. Furthermore, the proof of Lemma 2.2 will imply that equality cannot hold in any other cases; cf. $\left(2.6_{n}\right)-\left(2.7_{n k}\right)$ and (3.5) below.

Lemma $2.2_{n}$. Strengthen (2.2) in Lemma $2.1_{n}$ to

$$
a \leqq a_{0} \leqq \cdots \leqq a_{n-1} \leqq b \text { and let } a_{n}=b .
$$

Then, on $\left[a, a_{0}\right]$,

(2.6n) $|x(t)| \leqq \int_{t}^{b}\left|x^{\prime}(s)\right| d s \leqq \int_{t}^{b}\left|x^{(n)}(s)\right|(s-t)^{n-1} d s /(n-1) !$ 
and, on $\left[a_{k}, a_{k+1}\right]$, for $k=0, \cdots, n-1$,

$$
\begin{aligned}
(n-2) !|x(t)| \leqq & (n-2) ! \int_{a_{0}}^{t}\left|x^{\prime}(s)\right| d s \\
\leqq & \sum_{j=0}^{k-1} C(n-2, j) \int_{a_{j}}^{a_{j+1}}(t-r)^{j} F_{j}(r) d r \\
& +C(n-2, k) \int_{a_{k}}^{t}(t-r)^{k} F_{k}(r) d r
\end{aligned}
$$

where

$$
F_{j}(r)=\int_{r}^{b}\left|x^{(n)}(s)\right|(s-r)^{n-2-j} d s .
$$

In particular, if $M=\max \left|x^{(n)}\right|$ on $[a, b]$, then

$$
\begin{aligned}
& n !|x(t)| \leqq M\left[(b-t)^{n}-\left(b-a_{0}\right)^{n}\right] \quad \text { on }\left[a, a_{0}\right], \\
& n !|x(t)| \leqq M\left[\left(b-a_{0}\right)^{n}-(b-t)^{n}\right] \max _{0 \leqq j \leqq k} C(n-1, j)
\end{aligned}
$$

on $\left[a_{k}, a_{k+1}\right]$ for $k=0,1, \cdots, n-1$.

Although the inequalities $\left(2.6_{n}\right),\left(2.7_{n k}\right)$ may not be in a very useful form, the point of these relations is that they have a simple proof and imply (2.8), (2.9) and Lemma 2.1 .

3. Proof of Lemma 2.2. On $\left(2.6_{n}\right)$. The relation $\left(2.6_{1}\right)$ is trivial on $\left[a, a_{0}\right]$. Assume $n \geqq 2$ and $\left(2.6_{n-1}\right)$. Thus $\left(2.6_{n-1}\right)$ applied to $x^{\prime}(t)$ gives

$$
\left|x^{\prime}(t)\right| \leqq \int_{t}^{b}\left|x^{(n)}(s)\right|(s-t)^{n-2} d s /(n-2) ! \quad \text { on }\left[a, a_{1}\right] .
$$

For $a \leqq t \leqq a_{0}$, the inequality

$$
|x(t)| \leqq \int_{t}^{a_{0}}\left|x^{\prime}(r)\right| d r \leqq \int_{t}^{b}\left|x^{\prime}(r)\right| d r
$$

combined with (3.1) gives $\left(2.6_{n}\right)$.

On (2.7 $\left.7_{n k}\right)$. The inequality (3.1) and $|x(t)| \leqq \int_{a_{0}}^{t}\left|x^{\prime}(r)\right| d r$ for $t \geqq a_{0}$ gives $\left(2.7_{n 0}\right)$ for $n=2,3, \cdots$ Assume $\left(2.7_{n k}\right)$ for fixed $k \geqq 0$ and all $n \geqq k+2$. We shall verify $\left(2.7_{n, k+1}\right)$. From $\left(2.7_{n k}\right)$ for $t=a_{k+1}$,

$$
\begin{aligned}
(n-2) ! \int_{a_{0}}^{a_{k+1}}\left|x^{\prime}(s)\right| & d s \\
& \leqq \sum_{j=0}^{k} C(n-2, j) \int_{a_{j}}^{a_{j+1}}\left(a_{k+1}-r\right)^{j} F_{j}(r) d r ;
\end{aligned}
$$

and $\left(2.7_{n-1, k}\right)$ applied to $x^{\prime}$ gives 


$$
\begin{aligned}
(n-3) !\left|x^{\prime}(v)\right| \leqq & \sum_{j=0}^{k-1} C(n-3, j) \int_{a_{j+1}}^{a_{j+2}}(v-r)^{j} F_{j+1}(r) d r \\
& +C(n-3, k) \int_{a_{k+1}}^{v}(v-r)^{k} F_{k+1}(r) d r
\end{aligned}
$$

for $a_{k+1} \leqq v \leqq a_{k+2}$ and $k=0, \cdots, n-2$. A quadrature of (3.3),

$$
\begin{aligned}
& (n-2) ! \int_{a_{k+1}}^{t}\left|x^{\prime}(v)\right| d v \\
& \leqq \\
& \quad \sum_{j=1}^{k} C(n-2, j) \int_{a_{j}}^{a_{j+1}}\left[(t-r)^{j}-\left(a_{k+1}-r\right)^{j}\right] F_{j}(r) d r \\
& \quad+C(n-2, k+1) \int_{a_{k+1}}^{t}\left(a_{k+1}-r\right)^{k+1} F_{k+1}(r) d r
\end{aligned}
$$

together with (3.2) gives $\left(2.7_{n, k+1}\right)$.

On (2.8). By (3.1), $\left|x^{\prime}(t)\right| \leqq M(b-t)^{n-1} /(n-1)$ ! on $\left[a, a_{1}\right]$. A quadrature over $\left[t, a_{0}\right]$ gives $(2.8)$.

On (2.9). The right side of $\left(2.7_{n k}\right)$ is not decreased if $\left|x^{(n)}(s)\right|$ is replaced by $M$ and, in the integrand, $t$ is replaced by $b$. Thus, on $\left[a_{k}, a_{k+1}\right]$,

$$
\begin{aligned}
n !|x(t)| \leqq & M \sum_{j=0}^{k-1} C(n-1, j)\left[\left(b-a_{j}\right)^{n}-\left(b-a_{j+1}\right)^{n}\right] \\
& +M C(n-1, k)\left[\left(b-a_{k}\right)^{n}-(b-t)^{n}\right] .
\end{aligned}
$$

This imples (2.9) and completes the proof of Lemma 2.2.

4. Proof of Corollary 2.1. The proof of $\left(2.7_{n k}\right)$ shows that if $a \leqq a_{0} \leqq a_{1}$ and $a_{k} \leqq t \leqq a_{k+1}$, then

$$
\begin{aligned}
n ! \int_{a_{0}}^{t}\left|x^{\prime}(s)\right| d s \leqq & M \sum_{j=0}^{k-1} C(n-1, j)\left[\left(b-a_{j}\right)^{n}-\left(b-a_{j+1}\right)^{n}\right] \\
& +M C(n-1, k)\left[\left(b-a_{k}\right)^{n}-(b-t)^{n}\right]
\end{aligned}
$$

whether or not $x\left(a_{0}\right)=0$. This inequality, with $a=a_{0}, k=n-1$, and $t=b=a_{n}$, implies (2.4), since $n ! C_{n}=\max C(n-1, j)$ for $0 \leqq j \leqq n-1$.

5. Proof of Theorem 1. Suppose that (1.1) has a solution $x(t) \neq 0$ with $n$ zeros, counting multiplicities, on $[a, b]$. It will be shown that the inequalities (1.8), (1.10), (1.11) and (1.12) cannot hold.

We can suppose that there are numbers

$$
a=a_{0} \leqq a_{1} \leqq \cdots \leqq a_{n-1}=b_{n-1} \leqq b_{n-2} \leqq \cdots \leqq b_{1} \leqq b_{0}=b,
$$


such that $x^{(k)}(t)=0$ if $t=a_{k}$ and $t=b_{k}$. Put $c=a_{n-1}=b_{n-1}, a<c<b$. The analogues of Lemma $2.1_{n-1}$ are applicable to each of the intervals $[a, c],[c, b]$. Actually, the analogues will be applied to $x^{(n-1-k)}$, rather than to $x$ with $n-1$ replaced by $k, k=1, \cdots, n-2$. Let $M=\max \left|x^{(n-1)}(t)\right|$ on $[a, c]$ and let $\left|x^{(n-1)}(r)\right|=M, a<r<c$.

Write (1.1) as

$$
\left(x^{(n-1)} \exp \int_{a}^{t} p_{0} d s\right)^{\prime}+\left(\exp \int_{a}^{t} p_{0} d s\right) \sum_{k=1}^{n-1} p_{k}(t) x^{(n-1-k)}=0 .
$$

An integration over the interval $[r, c]$ gives

$$
M \exp \int_{a}^{r} p_{0} d s<\sum_{k=1}^{n-1} \max _{[a, c]}\left|x^{(n-1-k)}\right| \int_{r}^{c}\left(\exp \int_{a}^{t} p_{0} d s\right)\left|p_{k}\right| d t .
$$

By Lemma $2.1_{k}$ and

$$
\exp \int_{r}^{t} p_{0} d s \leqq \exp \int_{a}^{c} p_{0}^{+} d s \quad \text { for } r \leqq t \leqq c,
$$

we get

$$
1<\left(\exp \int_{a}^{c} p_{0}^{+} d s\right) \sum_{k=1}^{n-1} C_{k}(c-a)^{k} \int_{a}^{c}\left|p_{k}\right| d t
$$

Similarly,

$$
1<\left(\exp \int_{c}^{b} p_{0}^{-} d s\right) \sum_{k=1}^{n-1} C_{k}(b-c)^{k} \int_{c}^{b}\left|p_{k}\right| d t
$$

On (1.12). Since either $c \leqq(a+b) / 2$ or $c \geqq(a+b) / 2$, that is, $c-a \leqq(b-a) / 2$ or $b-c \leqq(b-a) / 2,(5.1)$ and (5.2) imply that either $Q_{1}>1$ or $Q_{2}>1$.

On (1.8). By (5.1) and (5.2), we have

$$
\begin{gathered}
1<\left(\exp \left\|p_{0}\right\|\right) \sum_{j=1}^{n-1} \sum_{k=1}^{n-1} C_{j} C_{k}(c-a)^{k}(b-c)^{j} \\
\cdot \int_{a}^{c}\left|p_{k}\right| d t \int_{c}^{b}\left|p_{j}\right| d t .
\end{gathered}
$$

Note that

$$
(c-a)^{k}(b-c)^{j} \leqq|I|^{k+j} k^{k} j^{j} /(k+j)^{k+j} \quad \text { for } a \leqq c \leqq b
$$

If $m \geqq 0$, then $j k /(j+k)^{2} \leqq 1 / 4$ implies that 


$$
\begin{aligned}
j^{j} k^{k} /(j+k)^{j+k} & =\left[j k /(j+k)^{2}\right]^{m} j^{j-m} k^{k-m} /(j+k)^{j+k-2 m} \\
& \leqq 2^{-2 m}[j /(j+k)]^{j-m}[k /(j+k)]^{k-m} .
\end{aligned}
$$

If $j, k \geqq i$ and $j, k \geqq m \geqq 0$, then

$$
j^{j} k^{k} /(j+k)^{j+k} \leqq 2^{-2 m}[j /(j+i)]^{j-m}[k /(k+i)]^{k-m} .
$$

The inequalities (5.3), (5.4) and (5.6), with $i=m=1$, show that

$$
\begin{aligned}
1 & <\left(\exp \left\|p_{0}\right\|\right)\left\{\sum_{k=1}^{n-1}\left(C_{k} / 2\right)[k /(k+1)]^{k-1}|I|^{k} \int_{a}^{c}\left|p_{k}\right| d t\right\} \\
& \times\left\{\sum_{j=1}^{n-1}\left(C_{j} / 2\right)[j /(j+1)]^{j-1}|I|^{j} \int_{c}^{b}\left|p_{j}\right| d t\right\} .
\end{aligned}
$$

Take the square root of both sides of this inequality and use the arithmetic-geometric mean inequality, applied to the last two factors. to obtain

$$
1<\left(\exp \frac{1}{2}\left\|p_{0}\right\|\right) \sum_{k=1}^{n-1}\left(C_{k} / 4\right)[k /(k+1)]^{k-1}|I|^{k} \int_{a}^{b}\left|p_{k}\right| d t
$$

On (1.10). This is proved in the same way using $i=m$ in (5.6).

On (1.11). Insert the inequality (5.4) into (5.3), interchange $j$ and $k$, and add the resulting inequalities to obtain

$$
\begin{aligned}
2< & \left(\exp _{\|}\left\|p_{0}\right\|\right) \sum_{j=1}^{n-1} \sum_{k=1}^{n-1} C_{j} C_{k} j^{j} k^{k}(j+k)^{-j-k}|I|^{j+k} \\
& \cdot\left\{\int_{a}^{c}\left|p_{k}\right| d t \int_{c}^{b}\left|p_{j}\right| d t+\int_{c}^{b}\left|p_{k}\right| d t \int_{a}^{c}\left|p_{j}\right| d t\right\} .
\end{aligned}
$$

The factors $\{\cdots\}$ satisfy

$$
\{\cdots\} \leqq\left\|p_{j}\right\| \cdot\left\|p_{k}\right\| \quad \text { for all } j, k \text {; }
$$

but if $j=k$,

$$
\{\cdots\}=2 \int_{a}^{c}\left|p_{k}\right| d t \int_{c}^{b}\left|p_{k}\right| d t \leqq \frac{1}{2}\left(\| p_{k}||\right)^{2} \quad \text { for } j=k .
$$

The last three formula lines contradict (1.11) and complete the proof of Theorem 1.

\section{REFERENCES}

0. A. M. Fink and D. F. St. Mary, On an inequality of Nehari, Proc. Amer. Math. Soc. 21 (1969), 640-642.

1. P. Hartman, Ordinary differential equations, Wiley, New York, 1964. MR 30 $\# 1270$. 
2. M. Hukuhara, On the zeros of solutions of linear ordinary differential equations, Súgaku 15 (1963), 108-109. (Japanese) MR 29\#3704.

3. A. J. Levin, Some estimates for a differentiable function, Dokl. Akad. Nauk SSSR 138 (1961), 37-38 = Soviet Math. Dokl. 2 (1961), 523-524. MR 23\#A3310.

4. - $A$ bound for a function with monotonely distributed zeros of successive derivatives, Mat. Sb. 64 (106) (1964), 396-409. (Russian) MR $29 \# 4941$.

5. - On linear second-order differential equations, Dokl. Akad. Nauk SSSR 153 (1963), 1257-1260= Soviet Math. Dokl. 4 (1963), 1814-1817. MR 28 \#2278.

6. Z. Nehari, On an inequality of Lyapunov, Studies in Mathematical Analysis and Related Topics, Stanford Univ. Press, Stanford, Calif., 1962, pp. 256-251. MR 26\#2684.

7. G. S. ZaYceva, A multipoint boundary value problem, Dokl. Akad. Nauk. SSSR 176 (1967), 763-765 = Soviet Math. Dokl. 8 (1967), 1183-1185. MR 36\#2874.

The Johns Hopkins University 